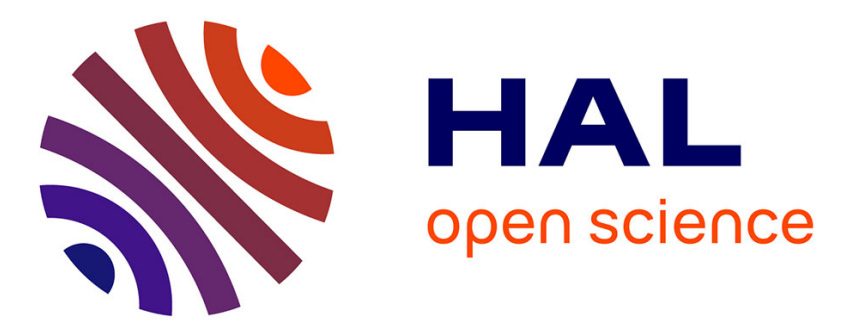

\title{
Why antiplectic metachronal cilia waves are optimal to transport bronchial mucus
}

\author{
Sylvain Chateau, Julien Favier, Sébastien Poncet, Umberto d'Ortona
}

\section{To cite this version:}

Sylvain Chateau, Julien Favier, Sébastien Poncet, Umberto d'Ortona. Why antiplectic metachronal cilia waves are optimal to transport bronchial mucus. Physical Review E , 2019, 100 (4), pp.042405. 10.1103/PhysRevE.100.042405 . hal-02468006

\section{HAL Id: hal-02468006 https://hal.science/hal-02468006}

Submitted on 31 Aug 2020

HAL is a multi-disciplinary open access archive for the deposit and dissemination of scientific research documents, whether they are published or not. The documents may come from teaching and research institutions in France or abroad, or from public or private research centers.
L'archive ouverte pluridisciplinaire $\mathbf{H A L}$, est destinée au dépôt et à la diffusion de documents scientifiques de niveau recherche, publiés ou non, émanant des établissements d'enseignement et de recherche français ou étrangers, des laboratoires publics ou privés. 


\title{
Why antiplectic metachronal cilia waves are optimal to transport bronchial mucus
}

\author{
S. Chateau $\odot,{ }^{1,2}$ J. Favier, ${ }^{1, *}$ S. Poncet, ${ }^{1,2}$ and U. D'Ortona ${ }^{1}$ \\ ${ }^{1}$ Aix Marseille Univ, CNRS, Centrale Marseille, M2P2, Marseille, France \\ ${ }^{2}$ Université de Sherbrooke, Département de génie mécanique, Sherbrooke, (QC) J1K 2R1, Canada
}

(Received 4 February 2019; published 4 October 2019)

\begin{abstract}
The coordinated beating of epithelial cilia in human lungs is a fascinating problem from the hydrodynamics perspective. The phase lag between neighboring cilia is able to generate collective cilia motions, known as metachronal waves. Different kinds of waves can occur, antiplectic or symplectic, depending on the direction of the wave with respect to the flow direction. It is shown here, using a coupled lattice Boltzmann-immersed boundary solver, that the key mechanism responsible for their transport efficiency is a blowing-suction effect that displaces the interface between the periciliary liquid and the mucus phase. The contribution of this mechanism on the average flow generated by the cilia is compared to the contribution of the lubrication effect. The results reveal that the interface displacement is the main mechanism responsible for the better efficiency of antiplectic metachronal waves over symplectic ones to transport bronchial mucus. The conclusions drawn here can be extended to any two-layer fluid configuration having different viscosities, and put into motion by cilia-shaped or comb-plate structures, having a back-and-forth motion with phase lags.
\end{abstract}

DOI: 10.1103/PhysRevE.100.042405

\section{INTRODUCTION}

Many living organisms use cytoplasmic extensions, known as cilia or flagella, to generate propulsion. In mammals, cilia play an important role in a wide variety of biological processes [1,2], for instance in the displacement of nutrients in the cerebrospinal fluid [3], in the embryonic development by determining the left-right asymmetry of the heart [4], or in the transport of mucus in human lungs [5]. The latter configuration is driven by the so-called mucociliary clearance (MCC), which constitutes the core of the present paper.

MCC is the main defense mechanism developed by human body to protect itself against foreign particles (dusts, pollutants, allergens, bacterias, etc.) inhaled during the breathing cycle. To get rid of these particles, the airways surface liquid (ASL), whose main purposes are to act as a barrier and capture foreign particles, covers the surface of the upper respiratory tract. MCC thus consists in the transport and elimination of the ASL up to the throat where it is swallowed and then digested in the stomach.

As many biofluids, the ASL exhibits complex properties, mainly due to the presence of mucins which are macromolecules highly concentrated in sugar. However, the distribution of mucins is not spatially homogeneous [6]. As a result, the ASL is generally considered as being the superposition of two distinct fluid layers: The periciliary liquid (PCL) and the mucus phase above it. The 6-7 $\mu \mathrm{m}$ depth PCL layer is a fluid mainly composed of water [7] and generally considered as a Newtonian fluid. It acts as a lubricant for the mucus layer, allowing the latter to easily slip onto it. However, the mucus is characterized by a higher concentration of mucins $(\sim 2 \%)$, which results in a higher viscosity and a highly

\footnotetext{
*julien.favier@univ-amu.fr
}

non-Newtonian behavior [6]. Mucus has many complex rheological properties, such as shear-thinning, thixotropy, or viscoelasticity. However, its rheological properties are hard to capture due to their huge variability [8]. The depth of the mucus layer varies between 5-10 $\mu \mathrm{m}$ in the upper respiratory tract [9], but can reach up to $70 \mu \mathrm{m}$ in disease conditions [10]. Its flow is not uniform along the respiratory tract, nevertheless a typical value of $40 \mu \mathrm{m} \cdot \mathrm{s}^{-1}$ is reported in Ref. [11].

To induce mucus transport, billions of cilia cover the bronchial epithelium, by tufts of 200 to 300 cilia per cell, leading to a quite high density of around $5-8 \mathrm{cilia} / \mu \mathrm{m}^{2}$ [2]. To escape Purcell's scallop theorem [12,13] and be able to generate fluid propulsion [14], their beating pattern is composed of a fast stroke phase where the cilia are rigid and orthogonal to the flow, and a slower recovery phase where the cilia are bent. Cilia have a high aspect ratio between their length $(L \approx 7 \mu \mathrm{m})$ and diameter $(D \approx 0.3 \mu \mathrm{m})$, and beat at a frequency estimated between 10 and $20 \mathrm{~Hz}$ [2]. Note that the cilia tips can reach the mucus during the stroke phase [10].

Metachronal waves (MCW) are often observed in nature in large arrays of cilia, such as on the surface of Paramecium and Opalina $[15,16]$, or on the respiratory tissues of vertebrates $[2,17]$. The waves are termed "antiplectic" if the phase lag $\Delta \Phi$ between two cilia is positive and "symplectic" if $\Delta \Phi$ is negative. Antiplectic MCW move in the direction opposite to the flow generated by the cilia, while symplectic MCW move in the same direction. MCW have generated a large corpus of both theoretical [18-31] and experimental [15,32-35] research efforts. Antiplectic MCW have been observed to be more efficient to transport fluids than symplectic MCW $[18,23,24,26,27,29-31]$, but the physical mechanism underlying this mechanical efficiency is still an open question.

As proposed in our previous work [30], the efficiency refers to the maximization of the mean displacement in the $x$ direction during a beating cycle, divided by the mean power 
$P^{*}$ spent during this beating cycle: $\eta=\left(\left\langle d_{x}\right\rangle / L\right) / P^{*}$. We also performed a thorough parametric study on the transport and mixing capabilities of both antipleptic and symplectic MCW, by considering carpets of cilia equally distributed on a $(x, y)$ plane $[30,31]$. We observed that antiplectic MCW were more efficient to transport the fluids over a large range of Reynolds numbers. However, we could not conclude regarding the origin of this better efficiency. To our knowledge, only Refs. [22,27,28] worked on explaining the physical mechanism responsible for the better efficiency of $\mathrm{MCW}$ to transport fluids. Reference [27] proposed an explanation based on the vortex organization, while Ref. [22] pointed out that $\mathrm{MCW}$ are more able to exert their full force on the flow compared to synchronously beating cilia. Finally, Ref. [28] advanced that the better efficiency of antiplectic MCW could be explained by the clusterized behavior of the cilia during the recovery stroke, as it reduces drag and backward flow. However, all these studies occurred in a single-layer fluid, and therefore their models could not highlight the entire spectrum of mechanisms associated with MCW in the airway's surface layer. In this work, the objective is to unravel the physical mechanism responsible for the superior efficiency of antiplectic waves in a two-phase fluid environment composed of the periciliary (PCL) and mucus layers. We highlight here a new phenomenon, based on the blowing or suction of the PCL-mucus interface, depending on the cilia metachrony. Additionally, the interface motion is found to create different systems of vortices for the antiplectic and symplectic MCW. This newly identified mechanism deforms the PCL-mucus interface where the lubrication effect, which allows the mucus to slip on the low-viscosity PCL layer, occurs. To understand how these two phenomena interact, we perform a comparative study of their respective contributions to the generated fluid velocity. The present results concern a large spectrum of applications involving any two-layer configuration having different viscosities in living bodies [36] or microchips flows [37], and also offer new perspectives on the understanding of respiratory diseases [38-40].

\section{NUMERICAL METHOD}

The Boltzmann equation describes the behavior of a gas from a microscopic point of view. The lattice Boltzmann method (LBM) solves the discrete Boltzmann equation for an ensemble of distribution functions $f_{i}(x, t)$ on a discrete lattice. These distribution functions describe the probability that ensembles of particles, with velocity $\mathbf{e}_{i}$, collide and then stream along the discrete velocity vectors $\mathbf{e}_{i}$. By doing a Chapman-Enskog analysis, one can recover the Navier-Stokes equations [41].

\section{Mathematical description}

\section{Single-component LB model}

In LBM, the fluid status is updated in time by resolving the discrete Boltzmann equation [42]:

$$
f_{i}\left(\mathbf{x}+\mathbf{e}_{i} \Delta t, t+\Delta t\right)=f_{i}(\mathbf{x}, t)-\frac{\Delta t}{\tau}\left[f_{i}(\mathbf{x}, t)-f_{i}^{(\mathrm{eq})}(\mathbf{x}, t)\right],
$$

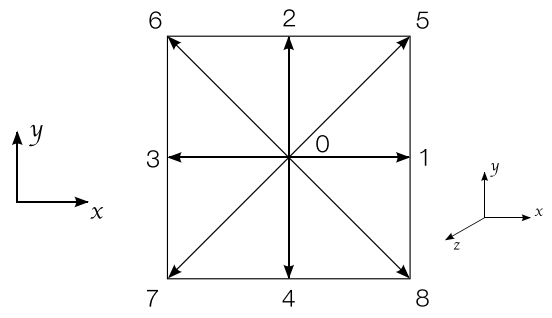

(a)

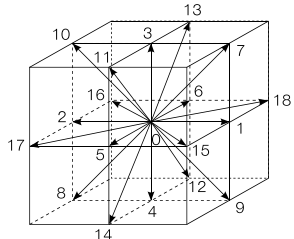

(b)
FIG. 1. Discrete velocities arrangement on a lattice cell: (a) D2Q9 lattice; (b) D3Q19 lattice.

where $f_{i}(\mathbf{x}, t)$ represents the distribution function at time $t$ and position $\mathbf{x}$ in the $i$ th direction of the lattice. These distribution functions move along a set of discrete velocity vectors $\mathbf{e}_{i}$ which depends on the lattice considered (Fig. 1). Here, Eq. (1) uses the single relaxation time (SRT) Bhatnagar-Gross-Krook (BGK) collision operator [43]. This model is based on a relaxation time $\tau$, which is linked to the lattice viscosity $v$ by $\tau=3 v+0.5$ using the classical normalization procedure, i.e., $\Delta x=\Delta t=1$ [41].

The local density $\rho$ and momentum $\rho \mathbf{u}$ at each lattice node can be obtained by summing all functions $f_{i}(\mathbf{x}, t)$ :

$$
\rho(\mathbf{x}, t)=\sum_{i=0}^{N} f_{i}(\mathbf{x}, t) \quad \rho \mathbf{u}(\mathbf{x}, t)=\sum_{i=0}^{N} f_{i}(\mathbf{x}, t) \mathbf{e}_{i},
$$

where $N$ is the number of discrete velocities on the lattice. The discrete equilibrium function $f_{i}^{\text {(eq) }}(\mathbf{x}, t)$ that appears in Eq. (1) can be obtained by Hermite series expansion of the Maxwell-Boltzmann equilibrium distribution [42]:

$$
f_{i}^{(\mathrm{eq})}=\rho \omega_{i}\left[1+\frac{\mathbf{e}_{i} \cdot \mathbf{u}}{c_{s}^{2}}+\frac{\left(\mathbf{e}_{i} \cdot \mathbf{u}\right)^{2}}{2 c_{s}^{4}}-\frac{\mathbf{u}^{2}}{2 c_{s}^{2}}\right],
$$

where $c_{s}=1 / \sqrt{3}$ is the speed of sound in lattice unit ("lu" hereafter). The weight coefficients $\omega_{i}$ are $\omega_{0}=4 / 9, \omega_{1-4}=$ $1 / 9$, and $\omega_{5-8}=1 / 36$ for D2Q9 lattices, and $\omega_{0}=1 / 3$, $\omega_{1-6}=1 / 18$, and $\omega_{7-18}=1 / 36$ for D3Q19 lattices [44].

Body force effects are introduced by adding an extra term to Eq. (1):

$$
\begin{aligned}
f_{i}\left(\mathbf{x}+\mathbf{e}_{i} \Delta t, t+\Delta t\right)= & f_{i}(\mathbf{x}, t)-\frac{\Delta t}{\tau}\left[f_{i}(\mathbf{x}, t)-f_{i}^{(\mathrm{eq})}(\mathbf{x}, t)\right] \\
& +\Delta t F_{i}(\mathbf{x}, t),
\end{aligned}
$$

where $F_{i}$ is given by the following equation:

$$
F_{i}=\left(1-\frac{\Delta t}{2 \tau}\right) \omega_{i}\left[\frac{\mathbf{e}_{i}-\mathbf{u}}{2 c_{s}^{2}}+\frac{\mathbf{e}_{i} \cdot \mathbf{u}}{c_{s}^{4}} \mathbf{e}_{i}\right] \cdot \mathbf{F} .
$$

Here, $\mathbf{F}$ represents the body force per unit volume. The macroscopic velocity $\mathbf{u}$ must then be updated in order for the system to recover the Navier-Stokes equation:

$$
\rho \mathbf{u}=\sum_{i} \mathbf{e}_{i} f_{i}+\frac{\Delta t}{2} \mathbf{F} .
$$




\section{Multicomponent LB model}

When considering two or more fluid components and body force effects, the discrete LB equation is written as follows:

$$
\begin{aligned}
f_{i}^{\sigma}\left(\mathbf{x}+\mathbf{e}_{i} \Delta t, t+\Delta t\right)= & f_{i}^{\sigma}(\mathbf{x}, t)+\Delta t F_{i}^{\sigma}(\mathbf{x}, t) \\
& -\frac{\Delta t}{\tau_{\sigma}}\left[f_{i}^{\sigma}(\mathbf{x}, t)-f_{i}^{\sigma(\mathrm{eq})}(\mathbf{x}, t)\right],
\end{aligned}
$$

where $f_{i}^{\sigma}(\mathbf{x}, t)$ and $\tau_{\sigma}$ are the distribution functions and the single relaxation time of the $\sigma$ th component, respectively. The expression of the equilibrium distribution function now reads

$$
f_{i}^{\sigma(\mathrm{eq})}=\rho_{\sigma} \omega_{i}\left[1+\frac{\mathbf{e}_{i} \cdot \mathbf{u}_{\sigma}^{(\mathrm{eq})}}{c_{s}^{2}}+\frac{\left(\mathbf{e}_{i} \cdot \mathbf{u}_{\sigma}^{(\mathrm{eq})}\right)^{2}}{2 c_{s}^{4}}-\frac{\mathbf{u}_{\sigma}^{(\mathrm{eq})} \cdot \mathbf{u}_{\sigma}^{(\mathrm{eq})}}{2 c_{s}^{2}}\right],
$$

where $\rho_{\sigma}$ and $\mathbf{u}_{\sigma}^{(\mathrm{eq})}$ are the density and equilibrium velocity of the $\sigma$ th component, respectively. In the model of Ref. [47], the equilibrium velocity $\mathbf{u}_{\sigma}^{(\mathrm{eq})}$ is identical for the two fluid components. The expressions of $\rho_{\sigma}$ and $\mathbf{u}_{\sigma}^{(\mathrm{eq})} \mathrm{read}$

$$
\rho_{\sigma}=\sum_{i} f_{i}^{\sigma} \quad \mathbf{u}_{\sigma}^{(\mathrm{eq})}=\mathbf{u}^{*}=\frac{\sum_{\sigma} \sum_{i} \mathbf{e}_{i} f_{i}^{\sigma} / \tau_{\sigma}}{\sum_{\sigma} \sum_{i} f_{i}^{\sigma} / \tau_{\sigma}} .
$$

The explicit forcing term $F_{i}^{\sigma}$ in Eq. (7) is linked to the total body force $\mathbf{F}_{\sigma}$ per unit volume exerted on the $\sigma$ th component:

$$
F_{i}^{\sigma}=\left(1-\frac{\Delta t}{\tau_{\sigma}}\right) \frac{\mathbf{F}_{\sigma} \cdot\left(\mathbf{e}_{i}-\mathbf{u}_{\sigma}^{(\mathrm{eq})}\right)}{\rho_{\sigma} c_{s}^{2}} f_{i}^{\sigma(\mathrm{eq})} .
$$

Based on the methodology of Ref. [45], one can add a ShanChen-type fluid-fluid cohesion force $\mathbf{F}_{\sigma}^{\mathrm{SC}}$ in the total body force vector $\mathbf{F}_{\sigma}$ to model the two-component behavior. The expression of the Shan-Chen type fluid-fluid cohesion force is [46]

$$
\mathbf{F}_{\sigma}^{\mathrm{SC}}(\mathbf{x}, t)=-G_{\mathrm{coh}} \rho_{\sigma}(\mathbf{x}, t) \sum_{i} \omega_{i} \rho_{\sigma^{\prime}}\left(\mathbf{x}+\mathbf{e}_{i} \Delta t, t\right) \mathbf{e}_{i},
$$

where $\sigma^{\prime}$ represents a fluid different from $\sigma$; and where $G_{\text {coh }}$ is a parameter that controls both the fluid immiscibility and surface tension. Thus, $G_{\text {coh }}$ must be chosen high enough so that a sharp interface between the fluids is maintained at all times. Finally, note that with a Shan-Chen-type fluid-fluid cohesion force, the interface motion is captured in a natural way since the force is directly added to the fluid equations, and there is no discontinuity of the fluid velocity at the interface.

\section{The immersed boundary method}

The aim of the immersed boundary (IB) method is to impose velocity boundary conditions on the Eulerian fluid nodes that surround a solid, by adding an extra body force $\mathbf{F}_{\sigma}^{\mathrm{IB}}$ to the fluid equations, so that the macroscopic fluid velocity can equal the velocity at the Lagrangian points modeling the solid boundary. Hence, an IB force $\mathbf{F}_{\sigma}^{\mathrm{IB}}$ is also included in the total body force vector $\mathbf{F}_{\sigma}$ of Eq. (10), so that $\mathbf{F}_{\sigma}=\mathbf{F}_{\sigma}^{\mathrm{IB}}+\mathbf{F}_{\sigma}^{\mathrm{SC}}$. The macroscopic velocity $\mathbf{u}_{\sigma}$ given by Ref. [47] writes

$$
\rho_{\sigma} \mathbf{u}_{\sigma}=\sum_{i} \mathbf{e}_{i} f_{i}^{\sigma}+\frac{\Delta t}{2} \mathbf{F}_{\sigma}
$$

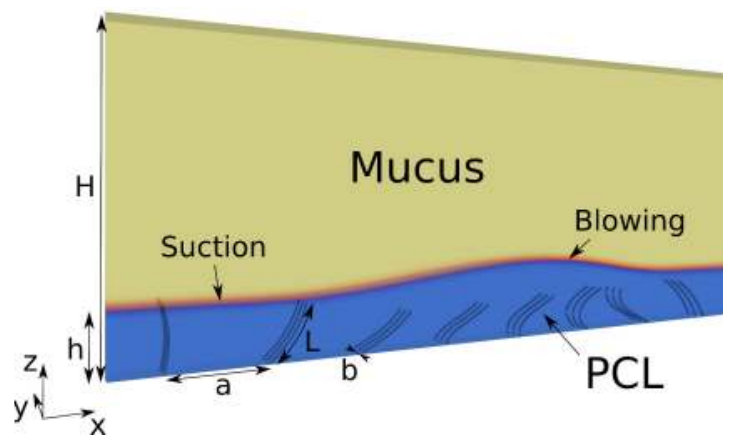

FIG. 2. Schematic view of the domain. The PCL phase is in blue and the mucus phase is in yellow. One can see the displacement of the PCL-mucus interface as a consequence of the hydrodynamic coupling between the fluid and solid motions.

The forcing term of the IB method is derived using a classical procedure which relies on two operators:

(1) The interpolation-In this step, the fluid velocity at the Eulerian nodes are used to perform an interpolation of the fluid velocity on the Lagrangian points.

(2) The spreading-An IB-related force is obtained as a function of the difference between the solid velocity and the interpolated fluid velocity. This force is spread to the surrounding Eulerian nodes to ensure the no-slip velocity condition at the fluid-solid boundary.

The present LBM-IB coupling has been validated in previous studies [48,49] and its accuracy was demonstrated on configurations involving complex and flexible immersed structures involving infinitely thin walls. For more details about the flow solver, the reader is referred to Refs. [48,49] and to Ref. [30] for the validation of the numerical results on the mucus transport.

\section{Geometrical modeling}

In the following, the fluid motions (mucus and PCL) are solved using the multicomponent LBM scheme previously described with a D3Q19 scheme. All variables are given in lattice units (lu) except when stated otherwise. Note that if a quantity is given without unit in the text, it is implied that it is expressed in lattice units. Both the PCL and the mucus are considered to be Newtonian fluids. The cilia motion is treated by the IB method. Periodic boundary conditions are imposed in the $x$ and $y$ directions, while no-slip and free-slip boundary conditions (BC) are used at the bottom and top walls, respectively. The computational domain is discretized using ( $N_{x}=385,7 \leqslant N_{y} \leqslant 41, N_{z}=54$ ) uniformly distributed lattice elements in the three directions of space, as shown in Fig. 2. The length $L$ of the cilia is set to 11 lattice units. The spacing between two cilia is set to $a=1.44 L$ in the $x$ direction, and $b$ varies from $0.18 L$ to $3.73 L$ in the $y$ direction. Hence, the number of cilia in the $x$ direction is $N_{\text {cil }}^{x}=24$ and remains constant in each simulation, while the number of cilia in the $y$ direction $N_{\text {cil }}^{y}$ may vary when modifying $b$ (for instance, a carpet of $24 \times 2$ cilia is considered for $b / L=$ $0.45)$. Their base point is located at $z=0$ which corresponds to the position of the epithelial surface. The beating period is $T_{\text {osc }}=N_{\text {it }} \Delta t$, where $N_{\text {it }}=5000$ is the number of iterations 
for performing a full beating cycle, and $\Delta t=1$ using the usual LBM normalization. The PCL fills the domain from $z=0$ up to an altitude $z=h$ which varies from $h=0.68 L$ to $h=1.64 L$. The wavelength of the imposed metachronal waves is $\lambda=34.9 L$ for the two phase lags $\Delta \Phi= \pm \pi / 12$, and $\lambda=11.6 L$ for the two phase lags $\Delta \Phi= \pm \pi / 4$. The viscosity of the mucus is fixed to $v_{\text {mucus }}=10^{-3} \mathrm{~m}^{2} \mathrm{~s}^{-1}$, and the viscosity ratio $r_{v}$ between the mucus and PCL is set to $r_{v}=10$, except stated otherwise. From a numerical point of view, the lattice viscosity for the mucus is $\nu_{\text {lat }}=0.338$, which leads to a relaxation time $\tau=1.51$. The lattice density is $\rho_{\text {lat }}=2$ for both the mucus and PCL.

The cilia motion is imposed to be in the $x$ direction only, since experimental observations showed that the stroke and recovery phases of respiratory cilia occur within the same plane [50,51]. Due to the intercilia spacing, no collisions between cilia occur during their beatings. The equations of the cilia motion are taken from Ref. [52] and reproduce a realistic $2 \mathrm{D}$ beating pattern similar to the one observed for real cilia by solving a 1D differential transport equation along a parametric curve:

$$
\frac{\partial P^{\prime}}{\partial t}+E(t) \frac{\partial P^{\prime}}{\partial \zeta}=0
$$

where $P(\zeta, t)$ denotes the position of the curve at time $t$ and at a normalized distance $\zeta=L_{S} / L$ where $L_{S}$ is the contour distance from the base point of a cilium, $E(t)=(\{1+$ $\left.\left.8 \cos ^{2}[\pi(t+0.25 T) / T]\right\} / T\right)$ is a term which mimics elastic effects, and $P^{\prime}=\partial_{\zeta} P$. With appropriate boundary conditions,

$$
\left\{\begin{array}{l}
P(0, t)=(0,0,0), \\
P^{\prime}(0, t)=\left[2 \cos \left(2 \pi t / T_{\mathrm{osc}}\right), 0, \cos \left(2 \pi t / T_{\mathrm{osc}}\right)\right],
\end{array}\right.
$$

a realistic beating pattern is obtained. In particular, the angular amplitude of this beating pattern is $\theta=2 \pi / 3$ as observed experimentally [2]. Note that here, the powerstroke is approximately as fast as the recovery stroke. The velocity $U_{\text {cil }}$ at the cilia tips can be computed by $U_{\text {cil }}=2 \theta L / T_{\text {osc }}$, and an oscillatory Reynolds number can be defined as $\operatorname{Re}^{\text {osc }}=U_{\text {cil }} L / v_{\text {muc }}$. In the following, results are presented for a Reynolds number of $\mathrm{Re}_{\mathrm{muc}}=10^{-2}$ in the mucus and $\mathrm{Re}_{\mathrm{PCL}}=10^{-1}$ in the PCL, thus no inertial effects occur in the simulations. Since the Stokes flow approximation prevails, the flow pattern remains identical even for a Reynolds number 100 times smaller as observed in human lungs. The interested reader is referred to Ref. [30], where the influence of the Reynolds number on the flow is discussed.

To quantify the flow produced by each kind of metachrony, the average velocity $U^{\text {av }}$ inside the whole domain during a beating cycle is computed. Its expression (in lu per time step) writes

$$
U^{\mathrm{av}}=\frac{1}{N_{x} N_{y} N_{z} T_{\mathrm{osc}}} \int_{0}^{T_{\mathrm{osc}}} \sum_{i, j, k} U_{i, j, k}(t) d t .
$$

The normalized average velocity $U^{*}$ is then defined as the average velocity $U^{\text {av }}$ divided by the maximal theoretical velocity $U_{\max }^{\mathrm{av}}=8 \times 10^{-3}$ reached by the cilia tips: $U^{*}=U^{\mathrm{av}} / U_{\max }^{\mathrm{av}}$. Then, both the PCL and mucus layers are taken into account in the computation of $U^{\text {av }}$. Such a choice is justified by the fact that it has been experimentally observed that PCL and mucus are transported at approximately the same rate; and that the PCL transport seems to depend on the presence of a mucus layer above it [7].

It is noteworthy that in the present model, a free-slip $\mathrm{BC}$ is used at the mucus-air interface, and thus a horizontal fluid velocity is imposed. This hypothesis has been carefully checked by simulating a three phase (PCL-mucus-air) configuration, using a very high viscosity ratio between mucus and air $\left(v_{\text {muc }} / \nu_{\text {air }}=1000\right)$, and it does not affect the results presented here. For the three-phase system, the mucus-air interface remains flat, and the flow pattern is identical to the two-phase system with a free-slip BC.

In the following, the "blowing-suction mechanism" will refer to the blowing or sucking of fluids due to the motion of cilia, whether there are one or two fluids. In the case of one fluid, this effect is used by pleurobrachia to improve the efficiency of their swimming movements [53]. Here, only twophase fluids are considered and the main effect of the blowingsuction is to move up and down the fluid-fluid interface. The "lubrication effect" refers to the increase (respectively, decrease) of $U^{\text {av }}$ that is only due to a diminution (respectively, increase) of the PCL viscosity.

\section{IDENTIFICATION OF THE PHYSICAL MECHANISM}

The flow patterns generated by the three metachrony are first considered for a value $h / L=0.91$ of PCL depth commonly given in the literature [7]. Figure 3(a) presents cilia during the stroke phase for the synchronous case (i.e., $\Delta \Phi=0)$. For such cilia motion, the PCL-mucus interface remains flat during the entire beating cycle. When the cilia perform their stroke phase, a strong flow is created inside the PCL layer, whereas a strong counter-flow is generated during the recovery phase, inducing on average a weak flow in the mucus [Fig. 3(a)]. Figure 3(b) displays the flow generated by an antiplectic MCW with $\Delta \Phi=\pi / 4$, and Fig. 3(c) the flow produced by a symplectic MCW with $\Delta \Phi=-\pi / 4$. In both Figs. 3(b) and 3(c), cilia 1 to 4 are in the stroke phase, and cilia 5 to 8 in the recovery phase. While the flow generated by the synchronous motion is parallel to the $x$ direction, it gets much more complex when metachrony is involved. Moreover, the velocity vectors reveal that the antiplectic MCW generates a much stronger flow than the synchronous case, and that the symplectic MCW is very inefficient to transport mucus due to the presence of recirculation cells. To go further, the normalized average fluid velocities $U^{*}$ produced by the three kinds of motion are compared. The symplectic MCW, the synchronous motion, and the antiplectic MCW produce a normalized average velocity $U^{*}$ equal to $-4.5 \times 10^{-2}, 2.3 \times 10^{-1}$, and $4.8 \times 10^{-1}$, respectively. The efficiency of the antiplectic $\mathrm{MCW}$ is obvious, as it produces a flow approximately 2 times larger than the synchronous case, while the symplectic MCW produces a small counterflow. Thus, one may wonder what is the fundamental difference between both kinds of motion to induce such different transport capabilities. This question, which has been addressed by many authors without a clear answer [18,23,24,26,27,29-31], is solved here.

The better efficiency of antiplectic MCW to transport mucus can be explained by the hydrodynamical interactions between neighboring cilia, which take the form of 


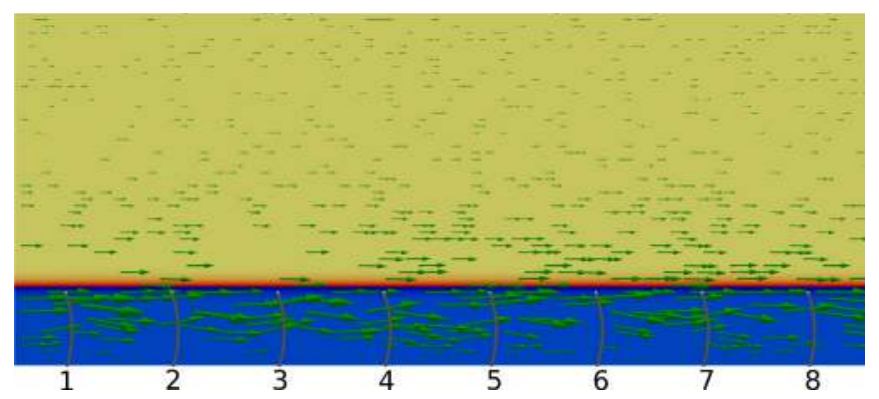

(a)

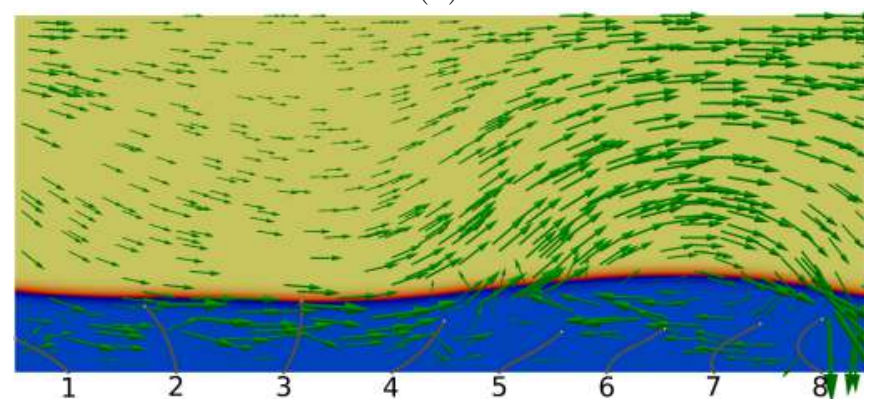

(b)

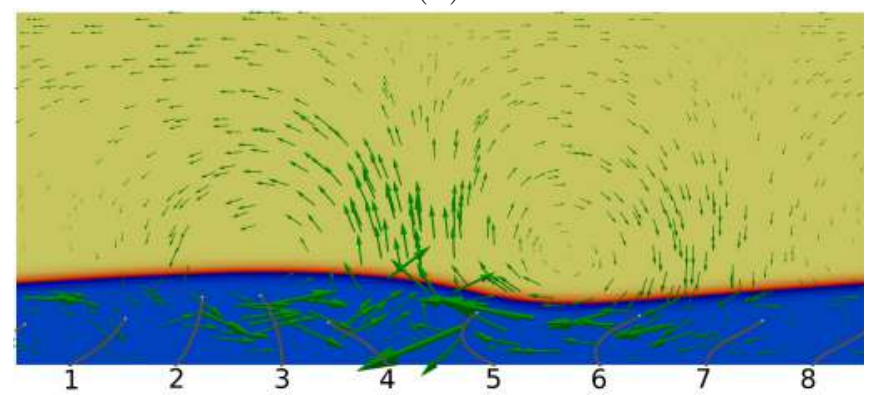

(c)

FIG. 3. Results obtained for $r_{v}=10, b / L=0.18$, and $h / L=$ 0.91 for (a) a synchronous wave $(\Delta \Phi=0)$; (b) an antiplectic MCW with $\Delta \Phi=\pi / 4$; and (c) a symplectic MCW with $\Delta \Phi=-\pi / 4$. Vectors indicate the flow propagation, and the same scale is used in each case. In these figures, for clarity purpose, only one-third of the computational domain is shown.

a blowing/suction mechanism occurring at the PCL-mucus interface. Indeed, for antiplectic MCW [Fig. 3(b)], cilia 1-4 are far away from each other during the stroke phase. Thus, an aspiration effect occurs onto the interface which is pushed downward, and allows the cilia to penetrate more deeply into the mucus. On the contrary, during the recovery phase, cilia 5-8 are closely packed, thus they blow the interface above them. Hence, their tips do not enter the mucus phase, and no reversal of the mucus flow occurs.

For symplectic MCW, the opposite phenomenon happens [Fig. 3(c)]. Cilia 5-8 are far away from each other during the recovery phase enabling the aspiration effect to occur and the cilia to penetrate the mucus layer. Thus, they create a greater reversal flow. During the stroke phase, cilia $1-4$ are closely packed, and the interface is now displaced above their tips so that the cilia do not penetrate the mucus during the stroke phase, thus minimizing their pushing effort.
Moreover, as shown on Figs. 3(b) and 3(c), different systems of vortices are created by the interface displacement for both kinds of metachrony. They are reported here as they can lead to an experimental validation of the proposed mechanism, using efficient optical techniques [54]. For antiplectic $\mathrm{MCW}$, only one vortex is created above the cilia during the recovery motion [see cilia 5-8 in Fig. 3(b)]. This is the direct consequence of the fact that cilia in the recovery motion do not penetrate the mucus: The reversal flow remains in the low viscosity PCL phase. On the contrary, the symplectic MCW generates a system of two neighboring vortices in the mucus [Fig. 3(c)], one rotating clockwise (above cilia 5-6) and the other one rotating anticlockwise (above cilia 2-3), as the cilia in the recovery phase generate a strong counterflow which cancels out the flow produced by the cilia in the stroke phase.

This new mechanism explains why antiplectic MCW are more efficient to transport fluids. It is worth mentioning that the presence of vortices in our simulations agrees particularly well with the experimental observations of [54], who observed a transition between a directional and a vortical flow in the region near the tips of embryonic cilia. Videos showing the blowing-suction of the PCL-mucus interface for different configurations are available online [55].

\section{PARAMETERS ACTING ON THE INTERFACE DISPLACEMENT}

The cilia spacing is the key parameter in the efficiency of this blowing-suction effect. Thus the evolution of $b / L$ is dissociated from $a / L$ (keeping $a / L=1.44$ to prevent cilia collisions), to highlight the fundamental mechanism. This configuration is relevant when a lack of cilia is observed as for severe asthma or other chronic respiratory diseases [38-40], or if we consider the spacings between two neighboring tufts of cilia. However, note that varying $a / L$ would also impact the blowing-suction mechanism. Figure 4 reports the influence of the cilia spacing $b / L$ in the direction perpendicular to the flow on the fluid velocity $U^{*}$. Symplectic and antiplectic MCW exhibit different behaviors: For antiplectic MCW, $U^{*}$ increases as the cilia spacing $b / L$ diminishes, while for

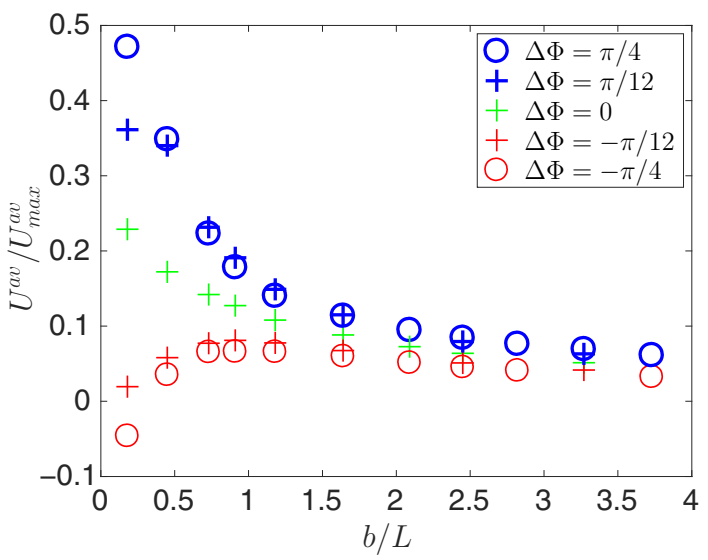

FIG. 4. Normalized average velocity $U^{*}=U^{\mathrm{av}} / U_{\max }^{\mathrm{av}}$ as a function of the cilia spacing $b / L$ in the $y$-direction for different phase lags $\Delta \Phi$. The value of $r_{v}$ is set to 10 , and the PCL depth to $h / L=0.91$. 
symplectic MCW, $U^{\text {av }}$ reaches a maximal value around $b / L \approx$ 1. Moreover, $U^{*}$ can even become negative (i.e., a flow reversal occurs) for the smallest value of $b / L$ tested (see $b / L=0.18$ for $\Delta \Phi=-\pi / 4$ in Fig. 4). For each value of $b / L$, the symplectic MCW generate a smaller average velocity than the synchronous case, and the synchronous case a smaller average velocity than the antiplectic MCW. Finally, it is noteworthy that for $b / L>1$, all kinds of synchronization tend to converge towards a plateau. Indeed, as $b / L$ increases, the blowing-suction mechanism becomes less efficient since the fluid can flow horizontally in between cilia instead of moving upwards and downwards. Therefore, the interface remains flat, allowing the cilia of each kind of metachrony to equally enter the mucus phase. Thus, for $b / L>1$, the small difference in the flow created by the antiplectic and symplectic MCW can only be due to the different torques exerted by the cilia during the stroke and recovery phases of each metachrony, as explained in Ref. [30]. Since the obtained velocities are almost identical, it shows that the difference in terms of efficiency between both kinds of MCW does not originate from the torques, but rather from the blowing-suction mechanism identified here. One can also observe that the trend of the synchronous motion is similar to the one of the antiplectic MCW. The PCL-mucus interface always remaining flat for cilia beating synchronously, the increase of $U^{*}$ for small $b / L$ ratios is due to the increase of the cilia density that inherently occurs when diminishing $b / L$. Hence, to compare only the effect of the blowing-suction of the interface on the flow generated by a given metachrony, one must look at the gain and loss in the average flow velocity $U^{*}$ compared to the synchronous case with the same cilia spacing $b / L$. For example, for $b / L=0.18$, the antiplectic MCW with $\Delta \Phi=\pi / 4$ generate an average flow $106 \%$ stronger than the synchronous case, while for the symplectic case, instead of the expected increase with $b / L$, the decrease is so strong that the average flow velocity becomes negative.

As the blowing-suction mechanism is moving the interface upwards or downwards, the PCL height has a crucial influence on the flow produced (Fig. 5). For very small phase lags $\Delta \Phi$, two neighboring cilia beat almost synchronously and their combined motion is similar to the one observed in the

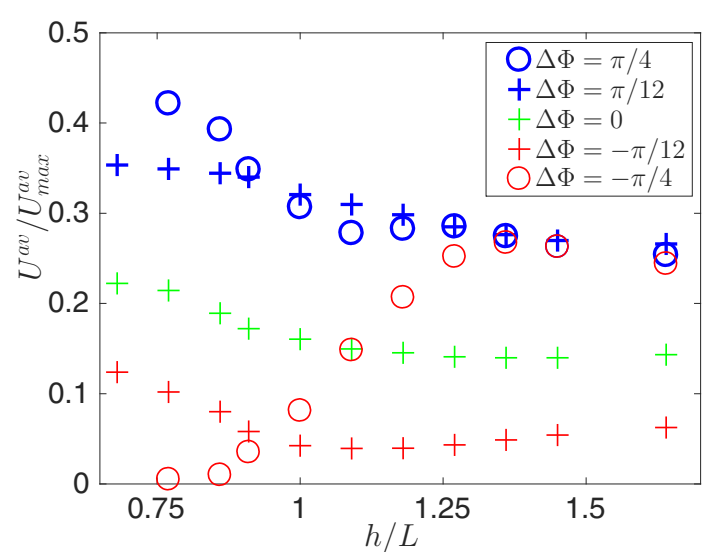

FIG. 5. Normalized average velocity $U^{*}=U^{\mathrm{av}} / U_{\max }^{\mathrm{av}}$ as a function of PCL depths $h / L$ for different phase lags $\Delta \Phi$. The value of $r_{v}$ is set to 10 , and $b / L$ to 0.45 . synchronous case (see $\Delta \Phi= \pm \pi / 12$ and $\Delta \Phi=0$ on Fig. 5). Furthermore, once the PCL depth becomes higher than 1, each metachrony reaches a plateau since the cilia are no longer able to penetrate the mucus layer. For larger phase lags, the results heavily depend on the kind of metachrony. Indeed, the behavior of the symplectic MCW for $\Delta \Phi=-\pi / 4$ differs from the case $\Delta \Phi=-\pi / 12$. This is a direct consequence of the interface motion. For $\Delta \Phi=-\pi / 4$, the blowing-suction effect is so powerful that for small $h / L$ values, the cilia can penetrate the mucus layer during the recovery phase, producing a strong counter-flow in the mucus. Then, as the PCL depth increases, the cilia are less and less able to reach the mucus, resulting in a steep increase of $U^{*}$ once the cilia tips can no longer penetrate into it during the recovery phase (see $h / L \geqslant 0.86$ for $\Delta \Phi=-\pi / 4$ in Fig. 5). This phenomenon underlines the importance of this blowing-suction mechanism highlighted here. Such depletion of the PCL layer is observed for patient suffering from cystic fibrosis [56]. Additionaly, it has been shown that mucus transport can not be achieved if the cilia penetrate the mucus layer during the recovery phase too [57].

At this point, it is worth noticing that the two waves with $\Delta \Phi= \pm \pi / 4$ induce an almost identical velocity when $h / L>$ 1.25 , contrary to the two waves with $\Delta \Phi= \pm \pi / 12$. For such values of $h / L$, the blowing-suction of the interface no longer occurs. It therefore strongly suggests the existence of another mechanism which happens when $h / L>1.25$. Preliminary results (not shown) tend to show that, for large values of $h / L$, the symplectic MCW increasingly become more efficient if the viscosity ratio is increased, or if the value of $\Delta \Phi$ is decreased. Further investigations are needed to understand the influence of these parameters.

Figure 6 displays the effect of the viscosity ratio $r_{v}$ on the averaged normalize velocity $U^{*}$ by acting on the viscosity of the PCL. Note that for the highest viscosity ratio tested, the Reynolds number in the PCL may increase up to unity. It has been shown in Ref. [30] that in this case the overall behavior of the flow is almost identical than for lower Reynolds numbers. Clearly, the viscosity ratio has a great influence on the fluid transport for each metachrony. For all

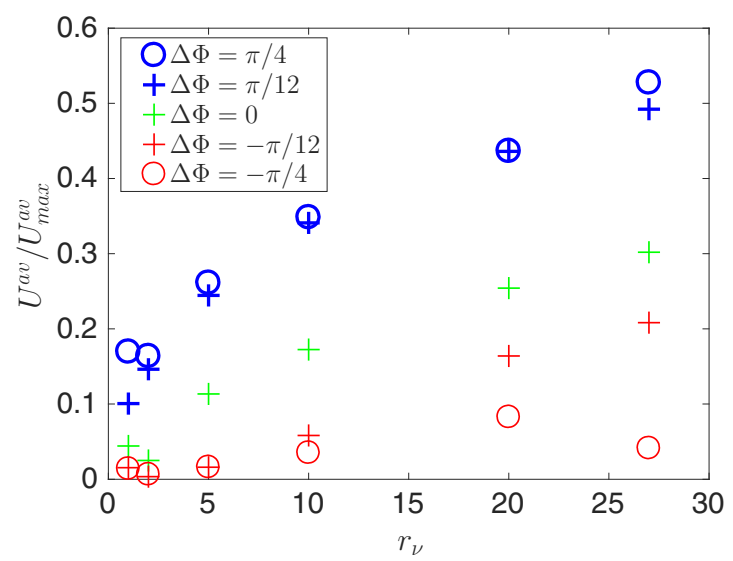

FIG. 6. Normalized average velocity $U^{*}=U^{\text {av }} / U_{\max }^{\mathrm{av}}$ as a function of the ratio of viscosity $r_{v}$ for different phase lags $\Delta \Phi$. The value of $b / L$ is set to 0.45 , and the PCL depth to $h / L=0.91$. 
values of $r_{v}$ tested, antiplectic MCW generate the largest fluid velocities. For the synchronous case $(\Delta \Phi=0)$, the increase in $U^{*}$ with $r_{v}>1$ is only due to the lubrication effect; indeed, the viscosity of the PCL is decreased to reach higher viscosity ratios, and it allows the mucus to slip onto the PCL more easily, leading to an increase of the average flow. However, the synchronous single-layer case (i.e., $\Delta \Phi=0$ and $r_{v}=1$ ) leads to a value of $U^{*}$ a little bit higher than the value obtained for $r_{v}=2$. The same is true for every other value of $\Delta \Phi$ tested, except for $\Delta \Phi=\pi / 12$. It shows that the advantages due to the lubrication effect start to appear only when the viscosity ratio is high enough $\left(r_{v}>2\right)$. Nevertheless, for each value of $r_{\nu}$, the difference in terms of flow velocity $U^{*}$ between the metachronal cases $\Delta \Phi \neq 0$ and the synchronous case $(\Delta \Phi=0)$ is only due to the blowing-suction mechanism. For the lowest viscosity ratio tested in a two-layers configuration (i.e., $r_{v}=2$ ), for which the effect of lubrication is the weakest, the blowing-suction mechanism allows the antiplectic MCW with $\Delta \Phi=\pi / 12$ to generate a flow 6 times stronger than the flow produced by the synchronous case. However, the synchronous case produces a much stronger flow than the symplectic MCW with $\Delta \Phi=-\pi / 12$, which induces a flow almost null. Similar results are obtained for the cases $\Delta \Phi=$ $\pm \pi / 4$. The effect remains important for the highest viscosity ratio tested: The antiplectic MCW with $\Delta \Phi=\pi / 12$ produces a flow 1.6 times stronger than the synchronous wave; and the synchronous wave a flow 1.5 times stronger than the symplectic MCW with $\Delta \Phi=-\pi / 12$. The same is true for the case $\Delta \Phi=\pi / 4$ for which the flow produced is close to the one generated by the case $\Delta \Phi=\pi / 12$ for each value of the viscosity ratio $r_{v}$; but not for the case $\Delta \Phi=-\pi / 4$ where a discrepancy with the case $\Delta \Phi=-\pi / 12$ appears when $r_{v}>10$. For the highest viscosity ratio tested, a decrease in $U^{*}$ is even observed for the case $\Delta \Phi=-\pi / 4$. While it is generally assumed that the PCL viscosity is similar to water, thus implying a huge viscosity ratio between the mucus and PCL layers, new experiments [56] tend to prove that the PCL is in fact much more viscous. This agrees well with the numerical results of [52] who found that the mucus transport was maximized for viscosity ratios ranging between 10 and 20 . This supports the applicability of the present results since for such viscosity ratio the blowing-suction of the interface is the main physical mechanism acting on the mucus transport.

\section{COMPETITION BETWEEN THE METACHRONAL GAIN AND THE LUBRICATION EFFECT}

As shown previously, the metachronal motion induces a blowing-suction mechanism which greatly influences the flow produced by the cilia, and could potentially enhance the flow in the case of antiplectic metachrony. However, as already mentionned in Sec. IV, another mechanism can also enhance the transport of mucus: The lubrication effect which also occurs at the PCL-mucus interface [58-60]. The aim of this part is thus to compare the respective contribution of the lubrication effect and metachronal gain on the average fluid velocity $U^{\text {av }}$ generated.

To do so, we make the hypothesis that the average fluid velocity $U^{\text {av }}$ can be expressed as the sum of two contributions:

$$
U^{\mathrm{av}}=U^{\mathrm{meta}}+U^{\text {lub }},
$$

where $U^{\text {meta }}$ is the part of $U^{\text {av }}$, which is due to the metachronal motion, and $U^{\text {lub }}$ the part due to the lubrication effect. Since the metachronal motion deforms the PCL-mucus interface, this hypothesis is correct only if the cross-effects between metachronal motion and lubrication are negligible. To estimate the effect of an interface modulation on lubrication, one could consider a Couette flow where the thickness of the layer $h$ is modulated by an amplitude $\delta h$. One can easily demonstrate that the correction in the shear-stress is of the order $(\delta h / h)^{2}$. As the maximal PCL-thickness variation is about $15 \%$ (see Fig. 3 for the largest cilia density $b / L=0.18$ ) and is lesser for all other cases, the effect of a thickness modulation to the lubrication is expected to be very weak. To estimate the influence of the viscosity ratio on the interface displacement, we have measured the interface modulation for two different viscosity ratios and see that the thickness modulations are almost identical [55]. A possible cross effect between metachronal motion and lubrication is then expected to remain small.

Several deductions can be made from Eq. (16). First, the metachronal gain is null for synchronously beating cilia, and it immediately follows from Eq. (16) that $U_{\Delta \Phi=0}^{\text {av }}=U_{\Delta \Phi=0}^{\text {lub }}$. The lubrication can be quantified by directly measuring the average velocity in the case of a flow without metachrony. Furthermore, as we just shown that the influence of an interface modulation is very weak on the lubrication effect, it follows that $U_{\Delta \Phi \neq 0}^{\text {lub }} \approx U_{\Delta \Phi=0}^{\text {lub }}$. While it is evident that the metachronal gain vanishes when the cilia beat synchronously (which leads to a flat interface), it is less clear when the lubrication effect vanishes. One may consider that when there is no more viscosity ratio $\left(r_{v}=1\right)$, the lubrication effect vanishes. However this is not the case with the present definition of the lubrication, $U^{\text {lub }}$ will vanish only when $r_{v}=0$. In the following, and to avoid any confusion, only situations where the lubrication effect is present $\left(r_{v}>1\right)$ will be considered.

Finally, let us remember that modifying the value of $b / L$ impacts the fluid velocity generated by the synchronous case (Fig. 4), because of the inherent change in cilia density. To get rid of this parasitic effect on the resulting fluid velocity, we divide Eq. (16) by the average velocity $U_{\Delta \Phi=0}^{\text {av }}$ generated by the synchronous case with the same cilia spacing $b / L$ and the same viscosity ratio $r_{v}$. By doing so, each term of Eq. (16) does not depend on the ciliary density. For the sake of clarity, we write "tilted" quantities, where " $\sim$ " refers to a velocity normalized by $U_{\Delta \Phi=0}^{\text {av }}$. It reads

$$
\tilde{U}^{\mathrm{meta}}=\tilde{U}^{\mathrm{av}}-\tilde{U}^{\mathrm{lub}} .
$$

In the following, only the antiplectic cases with $\Delta \Phi=$ $\pi / 12$ and $\pi / 4$ are considered. The case $\pi / 4$ leads to the strongest interface displacement. Indeed, the symplectic case with opposite phase lag $(\Delta \Phi=-\pi / 4)$ results in a counterflow for small values of $b / L$; and thus comparing the relative importance of two effects having opposite directions is not relevant.

Figure 7 presents the average velocity $\tilde{U}^{\text {meta }}$ as a function of $b / L$ for three values of the viscosity ratio $r_{\nu}$. Note that 


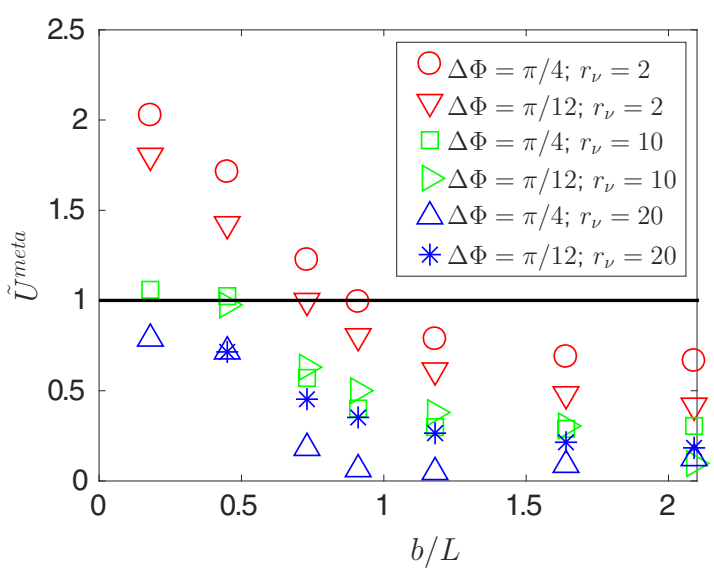

FIG. 7. Normalized average velocity $\tilde{U}^{\text {meta }}$ as a function of the cilia spacing $b / L$ in the $y$-direction for three values of the viscosity ratio $r_{v}$, for antiplectic metachronal waves with $\Delta \Phi=\pi / 4$ and $\Delta \Phi=\pi / 12$. The solid black line represents the value of $\tilde{U}^{\text {lub }}$. The value of the PCL depth is set to $h / L=0.91$.

$\tilde{U}^{\text {meta }}$ has been plotted only for values of $b / L \leqslant 2.1$, since the blowing-suction mechanism induced by metachronal motion can already be neglected for $b / L=2.1$, thus causing the metachronal gain to plateau. For the smallest viscosity ratio tested $\left(r_{v}=2\right)$, one can see on Fig. 7 that the metachronal gain is the dominant effect up to $b / L=1.91$. For the smallest value of $b / L$ tested $(b / L=0.18)$, the contribution of this mechanism on the average flow velocity is twice the contribution of the lubrication effect. However, when $r_{v}$ is increased $\left(r_{v}=10\right)$, one can notice that the contribution of the metachronal gain on the average fluid velocity strongly diminishes; and as soon as $b / L \geqslant 0.45$ the lubrication effect becomes dominant. For $r_{v}=20$, the lubrication effect contributes even more to the average fluid velocity produced than the metachronal gain for every values of $b / L$ tested. Nevertheless, the contribution on the average fluid velocity of the metachronal gain approaches the contribution of the lubrication effect when $b / L \leqslant 0.45$. Since for real respiratory epithelium the ciliary density is very high, it indicates that the metachronal gain can not be neglected.

The conclusions are valid for a phase lag $\Delta \Phi=\pi / 4$ which induces a strong displacement of the mucus-PCL interface. However, one may ask how the metachronal gain and lubrication mechanisms interact for other values of $\Delta \Phi$, and if there is a specific value of phase lag for which both phenomena act together in an optimal way. In particular, it is expected that smaller values of $\Delta \Phi$, for which the blowing-suction mechanism is weaker, would lead to a better lubrication. This is reported in Fig. 7 for a metachronal wave with $\Delta \Phi=\pi / 12$. One may note that the relative effect of the metachronal gain is decreased, but the difference with the case $\Delta \Phi=\pi / 4$ remains small. Inversely, one could expect that higher values of $\Delta \Phi$, for which the interface displacement is higher, will limit the importance of the lubrication effect. More data are thus required to fully characterise the interactions of these two mechanisms.

Finally, note that the respective contributions of these two mechanisms were only compared by looking at the average fluid velocity produced. The next steps towards a deeper understanding of the interplay of these two mechanisms consist in a study of the interface's shape, its amplitude, as well as the influence of a small ciliary disorganization on the interface displacement (as cilia in nature are not perfectly organized).

\section{CONCLUSION}

A blowing-suction effect occurring at the PCL-mucus interface has been identified as being the key mechanism explaining why antiplectic MCW are more efficient than symplectic or synchronous waves to transport fluids in a twolayer configuration. This mechanism allows the cilia in the stroke phase to better penetrate the mucus phase for antiplectic $\mathrm{MCW}$, while hindering them to reach the mucus during the recovery phase. The effect is even more important as the PCL depth and cilia spacing decrease. The relative effect of blowing-suction over lubrication increases while the viscosity ratio decreases. Without this mechanism, the clearance velocity of antiplectic and symplectic metachronies becomes similar. The small differences of flow velocity between antiplectic and symplectic MCW are then only due to the torques exerted by the cilia, as shown in Ref. [30]. The results presented here show that, in the range of viscosity ratios tested, the interface displacement induced by the metachronal motion has a similar or larger impact on the average flow velocity than the lubrication effect. The conclusions drawn here can be applied to any two-phase configurations with fluids having different viscosities, and put into motion by solids, ranging from slender cilia to rigid rods, regularly spaced or not, as long as they have a back-and-forth motion with $\Delta \Phi \neq 0$. This is particularly interesting for the design of microchips flows [37], where this blowing-suction effect can easily be tuned to obtain higher fluid transport by acting on the cilia spacing, the depth of the fluids, and others parameters such as the temporal asymmetry between the back-and-forth motion, or the viscosity ratio between the two fluids. Note that some authors consider the airways surface liquid as being a single-layer fluid having a gradient of concentration in mucins in the vertical direction [52]. This vision would not impact significantly the proposed blowing-suction mechanism, as regions of different mucin concentrations, hence different viscosities, would be displaced accordingly to the cilia motion. Finally, this mechanism also offers new perspectives in the understanding of how respiratory diseases affect the mucus transport, such as cystic fibrosis where the secreted mucus is too viscous [40], or asthma where the mucociliary clearance process is strongly altered, with less cilia, abnormal beating processes, and defects in their spatial organization and coordination $[38,39]$.

\section{ACKNOWLEDGMENTS}

The Natural Sciences and Engineering Research Council of Canada is acknowledged (discovery Grant No. RGPIN-201506512). This work was granted access to the HPC resources of Aix-Marseille Université (Equip@Meso (ANR-10-EPQX29-01)) of the program "Investissements d'Avenir" supervised by the Agence Nationale de la Recherche, and to the HPC resources of Compute Canada. 
[1] C. Brennen and H. Winet, Annu. Rev. Fluid Mech. 9, 339 (1977).

[2] M. A. Sleigh, J. R. Blake, and N. Liron, Am. Rev. Respir. Dis. 137, 726 (1988).

[3] R. Faubel, C. Westendorf, E. Bodenschatz, and G. Eichele, Science 353, 176 (2016).

[4] J. McGrath and M. Brueckner, Curr. Opin. Genet. Dev. 13, 385 (2003).

[5] M. A. Sleigh, Chest. 80, 791 (1981).

[6] S. K. Lai, Y. Y. Wang, D. Wirtz, and J. Hanes, Adv. Drug. Deliver. Rev. 61, 86 (2009).

[7] H. Matsui, B. R. Grubb, R. Tarran, S. H. Randell, J. T. Gatzy, C. W. Davis, and R. C. Boucher, Cell 95, 1005 (1998).

[8] O. Lafforgue, I. Seyssiecq-Guarante, S. Poncet, and J. Favier, J. Biomed. Mater. Res. Part A 106, 386 (2017).

[9] D. Yager, T. Cloutier, H. Feldman, J. Bastacky, J. M. Drazen, and R. D. Kamm, J. Appl. Physiol. 77, 2333 (1994).

[10] J. H. Widdicombe and J. G. Widdicombe, Resp. Physiol. 99, 3 (1995).

[11] D. J. Smith, E. A. Gaffney, and J. R. Blake, Respir. Physiol. Neurobiol. 163, 178 (2008).

[12] E. M. Purcell, Am. J. Phys. 45, 3 (1997).

[13] In a few words, Purcell's scallop theorem states that the average position of a micro-swimmer can not change over one period if the sequence of shapes displayed by the swimmer is identical when seen in reverse (so-called reciprocal motion). The rate at which the micro-swimmer performs its beating is irrelevant: A breaking of symmetry is thus required.

[14] S. N. Khaderi, M. G. H. M. Baltussen, P. D. Anderson, J. M. J. den Toonder, and P. R. Onck, Phys. Rev. E 82, 027302 (2010).

[15] D. R. Brumley, M. Polin, T. J. Pedley, and R. E. Goldstein, J. R. Soc. Interface 12, 20141358 (2015).

[16] N. Narematsu, R. Quek, K. H. Chiam, and Y. Iwadate, Cytoskeleton 72, 633 (2015).

[17] A. Yaghi and M. B. Dolovich, Cells 5, 40 (2016).

[18] J. R. Blake, J. Fluid Mech. 55, 1 (1972).

[19] Y. W. Kim and R. R. Netz, Phys. Rev. Lett. 96, 158101 (2006).

[20] S. M. Mitran, Comput. Struct. 85, 763 (2007).

[21] T. Niedermayer, B. Eckhardt, and P. Lenz, Chaos 18, 037128 (2008).

[22] J. Elgeti and G. Gompper, Proc. Natl. Acad. Sci. USA 110, 4470 (2013).

[23] J. Rodrigo Vélez-Cordero and E. Lauga, J. Non-Newtonian Fluid 199, 37 (2013).

[24] E. M. Gauger, M. T. Downton, and H. Stark, Eur. Phys. J. E 28, 231 (2009).

[25] P. G. Jayathilake, Z. Tan, D. V. Le, H. P. Lee, and B. C. Khoo, Comput. Fluids 67, 130 (2012).

[26] H. Guo, J. Nawroth, Y. Ding, and E. Kanso, Phys. Fluids 26, 091901 (2014)

[27] S. N. Khaderi, J. M. J. den Toonder, and P. R. Onck, J. Fluid Mech. 688, 44 (2011).

[28] N. Osterman and A. Vilfan, Proc. Natl. Acad. Sci. USA 108, 15727 (2011).

[29] Y. Ding, J. C. Nawroth, M. J. McFall-Ngai, and E. Kanso, J. Fluid Mech. 743, 124 (2014).

[30] S. Chateau, J. Favier, U. D'Ortona, and S. Poncet, J. Fluid Mech. 824, 931 (2017).

[31] S. Chateau, U. D'Ortona, S. Poncet, and J. Favier, Front. Physiol. 9, 161 (2018).
[32] S. L. Tamm, J. Cell Biol. Interface 55, 250 (1972).

[33] M. J. Sanderson and M. A. Sleigh, J. Cell Sci. 47, 331 (1981).

[34] M. Vilfan, A. Potočnik, B. Kavčič, N. Osterman, I. Poberaj, A. Vilfan, and D. Babič, Proc. Natl. Acad. Sci. USA 107, 1844 (2010).

[35] R. Di Leonardo, A. Búzás, L. Kelemen, G. Vizsnyiczai, L. Oroszi, and P. Ormos, Phys. Rev. Lett. 109, 034104 (2012).

[36] M. E. V. Johansson, H. Sjövall, and G. C. Hansson, Nat. Rev. Gastroenterol. Hepatol. 10, 352 (2013).

[37] C. Y. Chen, C. Y. Chen, C. Y. Lin, and Y. T. Hu, Lab. Chip 13, 2834 (2013).

[38] B. Thomas, A. Rutman, R. A. Hirst, P. Haldar, A. J. Wardlaw, J. Bankart, C. E. Brightling, and C. O'Callaghan, J. Allergy Clin. Immunol. 126, 722 (2010).

[39] L. A. Laitinen, M. Heino, A. Laitinen, T. Kava, and T. Haahtela, Am. Rev. Respir. Dis. 131, 599 (1985).

[40] R. C. Boucher, Annu. Rev. Med. Dis. 58, 157 (2007).

[41] T. Kruger, H. Kusumaatmaja, A. Kuzmin, O. Shardt, G. Silva, and E. M. Viggen, The Lattice Boltzmann Method (Springer International Publishing, Switzerland, 2017).

[42] S. Chen and G. D. Doolen, Annu. Rev. Fluid Mech. 30, 329 (1998).

[43] P. L. Bhatnagar, E. P. Gross, and M. Krook, Phys. Rev. 94, 511 (1954).

[44] Y. H. Qian, D. d'Humières, and P. Lallemand, Europhys. Lett. 17, 479 (1992).

[45] N. S. Martys and H. Chen, Phys. Rev. E 53, 743 (1996).

[46] X. Shan and H. Chen, Phys. Rev. E 49, 2941 (1994).

[47] M. L. Porter, E. T. Coon, Q. Kang, J. D. Moulton, and J. W. Carey, Phys. Rev. E 86, 036701 (2012).

[48] Z. Li, J. Favier, U. D’Ortona, and S. Poncet, J. Comput. Phys. 304, 424 (2016).

[49] J. Favier, A. Revell, and A. Pinelli, J. Comput. Phys. 261, 145 (2014).

[50] M. A. Chilvers and C. O'Callaghan, Thorax 55, 314 (2000).

[51] P. R. Sears, K. Thompson, M. R. Knowles, and C. W. Davis, Am. J. Physiol.: Lung Cell. Mol. Physiol. 304, L170 (2013).

[52] R. Chatelin and P. Poncet, J. Biomech. 49, 1772 (2016).

[53] A. Dauptain, J. Favier, and A. Bottaro, J. Fluid. Struct. 24, 1156 (2008).

[54] W. Supatto, S. E. Fraser, and J. Vermot, Biophys. J. 95, L29 (2008).

[55] See Supplemental Material at http://link.aps.org/supplemental/ 10.1103/PhysRevE.100.042405 for illustration of the blowingsuction effect for the three kinds of cilia motion (antiplectic, symplectic, and synchrone) with different values of $b / L$ and $h / L$.

[56] N. Derichs, B-J. Jin, Y. Song, W. E. Finkbeiner, and A. S. Verkman, FASEB J. 25, 2325 (2011).

[57] W. L. Lee, P. G. Jayathilake, Z. Tan, D.-V. Le, H. P. Lee, and B. C. Khoo, Comput. Fluids 49, 214 (2011).

[58] J. M. Zahm, D. Pierrot, S. Vaquez-Girod, C. Duvivier, M. King, and E. Puchelle, Biorheology 26, 747 (1989).

[59] M. R. Knowles and R. C. Boucher, J. Clin. Invest. 109, 571 (2002)

[60] W. H. Anderson, R. D. Coakley, B. Button, A. G. Henderson, K. L. Zeman, N. E. Alexis, D. B. Peden, E. R. Lazarowski, C. W. Davis, S. Bailey, F. Fuller, M. Almond, B. Qaqish, E. Bordonali, M. Rubinstein, W. D. Bennett, M. Kesimer, and R. C. Boucher, Am. J. Respir. Crit. Care Med. 192, 182 (2015). 